\title{
Hacia la consolidación de la Enfermería Universitaria
}

\section{Towards the consolidation of the nursing college}

Mtra. Ma. Dolores Zarza Arizmendi

Directora de la Escuela Nacional de Enfermería y Obstetricia UNAM

Me es muy grato colaborar en esta ocasión con la Revista Enfermería Universitaria, publicación emblemática de la Escuela Nacional de Enfermería y Obstetricia de la Universidad Nacional Autónoma de México, para la difusión del quehacer científico de la comunidad de nuestra Escuela. Es de suma importancia para mí compartir con nuestros lectores una breve semblanza del estado del arte de la ENEO y asimismo presentar los programas estratégicos para mi labor frente a la Dirección de esta dependencia universitaria para el periodo 20112015.

La ENEO tiene una trayectoria de importancia estratégica para el sistema de salud por su labor central de docencia. A través de esta actividad sustantiva se forman a un gran número de profesionales de Enfermería, de Especialistas de posgrado y principalmente a un gran número de Enfermeras en servicio que encuentran en la oferta educativa del Sistema Universidad Abierta y Educación a Distancia de la ENEO la posibilidad de un cre- cimiento vertical y la profesionalización de su práctica, función institucional en estas vertientes que mucho han contribuido y contribuyen al desarrollo de los servicios de Enfermería que necesita nuestro país.

En efecto, la ENEO tiene un gran prestigio por su labor académica, pero también lo tiene por su función de investigación, la que se realiza en la propia Escuela y la que se lleva a cabo en la Red de unidades de investigación, nichos de actividad incipiente, pero en algunos casos muy productiva que contribuyen al acervo de evidencia científica para guiar la práctica de Enfermería en diversos campos. La ENEO tiene aprecio y reconocimiento entre las instituciones educativas de Enfermería del país por su capacidad instalada y dinámica de servicios a los estudiantes y maestros. Es una dependencia de la UNAM que dispone de las instalaciones más modernas para apoyar el aprendizaje y la enseñanza de la carrera, que también tiene un gran reconocimiento por sus aportacio- nes en la FEMAFEE, en ALADEFE, Ceneval, en la Comisión Permanente de Enfermería de la Secretaría de Salud, en la Ops, OMS, en las Asociaciones y Colegios de la disciplina, con el Consejo de Salubridad General, y por la importante labor de colaboración que realiza con instituciones de salud y educativas, públicas y privadas, de atención a la salud y de asistencia social, con sectores de gobierno federal y estatal, y con instituciones especializadas de la sociedad civil, que encuentran en la ENEO a una COmunidad académica y escolar comprometida con la salud del pueblo de México.

La ENEO es la mejor institución de docencia e investigación en Enfermería que tiene México, es la Escuela que ha crecido y se ha desarrollado por la influencia que tiene la UNAM para impulsar el desempeño institucional, es la mejor Escuela por el gran compromiso que tienen sus maestros y trabajadores, por el trascendente servicio que ofrecen sus egresados, pero es una institución que debe trabajar más 
intensamente y con una clara idea de los estándares que tiene que alcanzar para mantenerse.

En este contexto, y en congruencia con la experiencia obtenida a lo largo de estos años, a continuación presento los programas estratégicos que he propuesto en mi Plan de Trabajo al frente de la Dirección de la ENEO para el periodo 20112015, cargo que me ha conferido la H. Junta de Gobierno de nuestra máxima casa de estudios. Lograr la concreción y resultados con base en este Plan es mi principal reto para encauzar el cómo hacerlo, con base en un trabajo colegiado, colectivo e incluyente.

El Plan de Trabajo, considera la situación actual de la ENEO por áreas académico administrativas, con los aciertos y los retos que hay que enfrentar, y enuncia acciones a incorporar en el marco de ocho estrategias generales que se corresponden con las concomitantes estrategias definidas en el Plan de Desarrollo Institucional, propuestas que deberán someterse a la discusión y al análisis en un ejercicio de planeación institucional

Estas estrategias generales son: 1. Fortalecimiento al ingreso, permanencia, aprovechamiento escolar y titulación. Para la operación de esta estrategia se plantean acciones de apoyo académico para promover el avance escolar y el éxito en los estudios, para mejorar el perfil de ingreso, asegurar el cumplimiento de los programas académicos, incorporar la Evaluación a todos niveles, el uso de tecnologías educativas, dominio de otros idiomas y la vinculación con alumnos e investigadores de otras universidades del país así como del extranjero. Las acciones abarcan el ingreso,

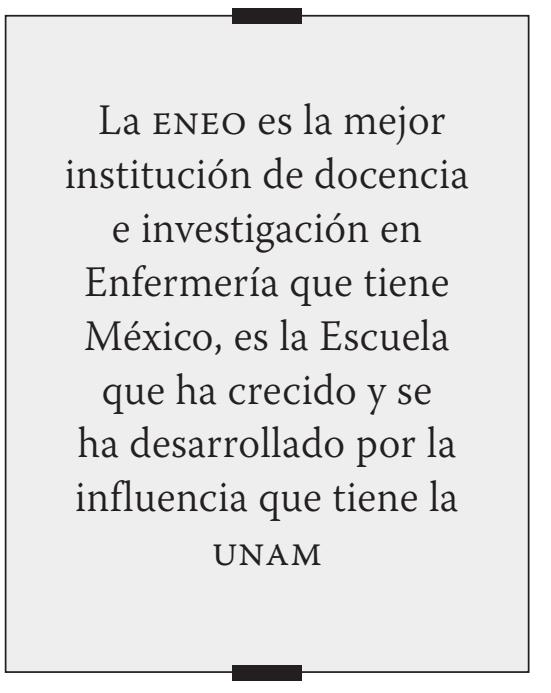

la permanencia, el aprovechamiento, la titulación, el servicio social y la incorporación temprana a programas de posgrado; 2. Consolidación del posgrado en enfermería. Se propone generar un programa de apoyo a la doble titulación con universidades del extranjero, así como iniciar un programa de formación de estudiantes sobresalientes que puedan transitar rápidamente a la maestría y al doctorado, vincular la actividad de investigación que realizamos en los Institutos Nacionales de Salud con la formación de posgrado, y promover el uso de las Tics (las tecnologías de la información y la comunicación) en la operación del posgrado con estrategias de educación a distancia; 3. Evaluación y desarrollo curricular. Crear el Departamento de Evaluación y Desarrollo Curricular como una unidad especializada en planeación educativa de pregrado y posgrado, adicionalmente, instrumentar un sistema integral de evaluación curricular que abarque acciones de evaluación y seguimiento de todos los planes de estudio y además generar un equipo de trabajo especializado en este departamento para enfrentar la acre- ditación y reacreditación de los planes de estudio; 4. Renovación e innovación de los procesos de enseñanza aprendizaje de la enfermería. Constituir equipos de trabajo en cada academia con capacidad para aplicar las Tics para que desarrollen cursos en línea para cada asignatura y materiales y medios educativos; 5. Fortalecimiento de la carrera académica. Realizar un análisis de la planta docente por figura y nivel en función de los proyectos institucionales para valorar la creación o transformación de nuevas plazas así como también fortalecer el programa de actualización y superación del personal académico y complementar el programa de formación y desarrollo de la planta docente con procesos que favorezcan la actualización constante del personal académico, también se propone adicionar como criterio para la evaluación de los docentes la opinión de los alumnos para el otorgamiento de estímulos a la productividad y de estímulos a la trayectoria académica; 6. Consolidación del sistema de investigación. Actualizar y reestructurar el comité interno de investigación, orientar líneas de interés con las Instituciones de Salud en la solución de problemas de la práctica profesional y especializada, buscar fuentes de financiamiento de apoyo sectorial para la actividad científica, identificar jóvenes académicos o becarios del posgrado con cualidades e interés en y para la investigación, orientar una generación de enfermeras al S.N.I, así como promover estancias en unidades de investigación de reconocida trayectoria en el país y en el extranjero; 7. Extensión académica y vinculación con la sociedad. 
Incrementar el número de aulas y dotar de los recursos académicos, equipamiento y apoyo administrativo al Centro de Educación Continua y Servicios de Enfermería Universitaria, instalar el sistema de videoconferencia interactiva y el apoyo en plataforma web en este centro y crear el comité académico de educación continua para ampliar la oferta de cursos en prácticas expertas; se propone también impulsar los cursos y diplomados de apoyo a la titulación y revisar la oferta de cursos postécnicos hacia nuevas orientaciones de desarrollo científico y tecnológico, diferentes a las que se operan en el posgrado; 8. Actualizar la estructura organizativa y reorientar la cultura organizacional. Hacer una selección cuidadosa del equipo directivo, que represente a los diferentes sectores y grupos de la escuela, con criterios bien definidos, así como ofrecer un curso de alta dirección a los funcionarios de la ENEO para iniciar una nueva etapa de avance institucional y establecer un programa anual de evaluación sobre el desempeño de los funcionarios en la actividad académico-administrativa para lograr esto se requiere crear la
Secretaría General Académica, con responsabilidades claramente diferenciadas en el vértice de la conducción institucional.

Por mi parte, aseguro que dispongo de la visión para reconocer los problemas y necesidades de la ENEO para este periodo, que cuento con el respaldo de un sector estratégico de la comunidad que a su vez también tiene capacidad para la función directiva, que tengo la sensibilidad para constituir un equipo directivo de todos los sectores de la Escuela para mantener la armonía interna, el reconocimiento a las capacidades de las personas y el respeto por las diferencias. Lo anterior porque al final siempre coincidimos en lo que hace falta, en lo que hay que mantener porque funciona, en lo que debemos corregir porque la experiencia nos señala que lo debemos hacer diferente, en lo que hay que generar para que nuestros estudiantes se atiendan mejor en sus necesidades de formación profesional y de posgrado, para que nuestros profesores dispongan de lo que hace falta para desempeñar con éxito sus tareas académicas y de las mejores condiciones para desarrollar su creati- vidad y capacidades para la investigación.

Estoy segura que todos quienes formamos parte de la comunidad de la ENEO queremos seguir sintiéndonos muy orgullosos de nuestra Escuela, satisfechos con sus instalaciones y respaldados por la rigurosidad académica de sus planes de estudio. Precisamente porque me siento muy comprometida con mi profesión, porque entiendo muy bien las dimensiones de la responsabilidad y las tareas que se comprenden en la función de la Dirección, estar al frente de esta encomiable labor constituye para mí una espléndida oportunidad para influir en el avance de la disciplina para la consolidación de la Enfermería Universitaria.

Finalmente, aprovecho esta oportunidad para reconocer a todo el equipo que integra la Revista "Enfermería Universitaria" y agradecer a todos nuestros entusiastas lectores, autores y revisores por apoyar esta importante publicación para continuar con el posicionamiento de la disciplina en el ámbito académico y de extensión. Enhorabuena. 